\title{
Fast collision resolution for real time services in SDMA based wireless ATM networks
}

\author{
Vornefeld, U.; Schieimer, D.; Walke, B.
}

Published in:

Vehicular Technology Conference, 1999 IEEE 49th

Link to article, DOI:

10.1109/VETEC.1999.780528

Publication date:

1999

Document Version

Publisher's PDF, also known as Version of record

Link back to DTU Orbit

Citation (APA):

Vornefeld, U., Schieimer, D., \& Walke, B. (1999). Fast collision resolution for real time services in SDMA based wireless ATM networks. In Vehicular Technology Conference, 1999 IEEE 49th IEEE.

https://doi.org/10.1109/VETEC.1999.780528

\section{General rights}

Copyright and moral rights for the publications made accessible in the public portal are retained by the authors and/or other copyright owners and it is a condition of accessing publications that users recognise and abide by the legal requirements associated with these rights.

- Users may download and print one copy of any publication from the public portal for the purpose of private study or research.

- You may not further distribute the material or use it for any profit-making activity or commercial gain

- You may freely distribute the URL identifying the publication in the public portal 


\title{
FAST COLLISION RESOLUTION FOR REAL TIME SERVICES IN SDMA BASED WIRELESS ATM NETWORKS *
}

\author{
Ulrich Vornefeld, Daniel Schleimer, Bernhard Walke \\ Communication Networks \\ Aachen University of Technology, Germany \\ e-mail: $\{$ ulvo|walke\}@comnets.rwth-aachen.de
}

\begin{abstract}
This paper considers random access and collision resolution algorithms in the context of Spatial Division Multiple Access (SDMA). We focus on the random access phase of HIPERLAN/2 systems currently under standardization at ETSI-BRAN. Based on the Dynamic Slot Assignment, DSA++ medium access control protocol, the influence of SDMA on a contention based access protocol is investigated under collision resolution schemes derived from classical splitting algorithms. Although this work is embedded in the framework of wireless ATM and HIPERLAN/2 systems, the ideas are generally applicable.
\end{abstract}

\section{INTRODUCTION}

One of the drawbacks of modern broadband wireless multimedia networks is the large amount of spectrum required. With respect to the progress of antenna technology and digital signal processing the deployment of SDMA (Spatial Division Multiple Access) seems to be a possible solution to increase the spectral efficiency. In this paper, we study how the deployment of SDMA affects contention based uplink transmissions in the scope of the Dynamic Slot Assignment, (DSA++) MAC protocol [1] for a WATM LAN corresponding to the HIPERLAN/2 system that is currently under standardization by the ETSI-Project Broadband $R a$ dio Access Networks (BRAN) [2]. Fig. 1 shows the basic frame structure of the protocol, which has been adopted to the current work assumptions of ETSI BRAN. Since the provision of Quality of Service (QoS) in terms of cell or packet loss and delay is of concern, fast collision resolution algorithms have to be applied. For delay sensitive services the throughput of the random access channel (RACH) is only of secondary interest. Starting with a classical binary splitting algorithm [3] we incorporate the benefits of SDMA into the collision resolution scheme [4] and develop and evaluate an adaptive algorithm that combines the advantages of enhanced signalling processing with MAC protocol control functions. The analytical models presented are parametrized using success probability functions obtained by simulation. Section II gives a short overview of the DSA++ protocol, section III addresses the influence of SDMA on splitting algorithms. In section IV the applied RACH model is presented, section V evaluates classical q-ary splitting in the context of SDMA and finally in section VI the newly developed adaptive splitting algorithm is introduced and throughput and delay are analyzed.

\section{GENERIC DSA++ MAC PROTOCOL}

The task of a medium access control protocol is to co-ordinate the wireless terminals' access to the shared radio channel. The

\footnotetext{
* Supported by: The German Federal Ministry of Education, Science, Research and Technology (Project: ATMmobil)
}

reservation based DSA++ MAC protocol grants transmission capacity to the terminals per slot to satisfy their current transmission capacity demands. The protocol is centrally controlled by the base station that operates a scheduler, which groups the various control and data channels into MAC frames of flexible structure and length. Therefore the wireless terminals have to transmit their capacity requests via the uplink to the base station. A complete MAC frame consists of a broadcast phase, a downlink phase, an uplink phase and a random access phase, Fig. 1. According to the current work assumptions of ETSI-BRAN the protocol differentiates the following channels:
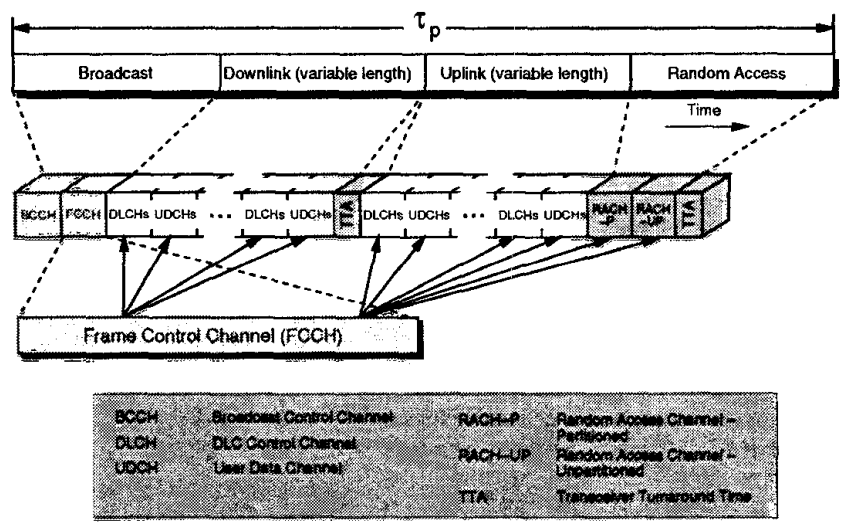

Figure 1: MAC frame of the DSA++ protocol

Broadcast Control Channel, BCCH: Conveys signalling information concerning the whole radio cell. Every MAC frame starts with the BCCH.

Frame Control Channel, FCCH: Transmits information about the structure of the MAC frame. It contains announcements and reservations of control and data channels the individual wireless terminals have to transmit in or to receive.

Data Link Control Channel, DLCH: Is associated to the UD$\mathrm{CH}$ and conveys signalling messages, such as acknowledgements and resource requests. The downlink phase as well as the uplink phase comprise DLCHs.

User Data Channel, UDCH: The UDCH serves to transmit user data (ATM cells) via up- and downlink.

Random Access Channel - Partitioned, RACH-P: A varying subset of associated wireless terminals is allowed to access this channel. 
Random Access Channel - Unpartitioned, RACH-UP: An uplink contention channel that can be accessed by any wireless terminal.

BRAN has also defined additional control channels: Slow Broadcast Channel (SBCH), Inband Channel (IBCH), and Dedicated Control Channel (DCCH), which are beyond the scope of this paper. To meet the QoS requirements especially of delay sensitive real time services, fast and in time signalling of capacity requests to the base station is necessary. The DSA++ protocol provides two methods for transmission of capacity requests to the base station via the uplink:

Explicit signalling using the DLCH: The base station scheduler reserves a slot for the DLCH to receive a terminal's capacity request message.

Random access using the RACH: The terminals can randomly access RACH slots to transmit their capacity request messages. Depending on the applied algorithm, capacity request messages usually are transmitted via the RACH-P.

At the end of each MAC frame the scheduler updates its information database about the terminal's capacity demands by evaluating the received request messages. This information together with the downlink transmission demands provide the input for the scheduling algorithm to determine the structure of the subsequent MAC frame. The base station broadcasts this structure via the FCCH to inform all the terminals about their individual transmission and reception schedule.

\section{SDMA AND RANDOM ACCESS}

SDMA enables the correct reception of $i$ out of $k$ simultaneously transmitting terminals within the same time slot at the same frequency by means of spatial filtering. However the number of concurrently receivable signals is limited by an upper bound $M$ determined by the antenna system and the spatial filtering algorithm [5]. To transmit their capacity request messages all terminals are allowed to access the RACH and since no additional restrictions are imposed on the access scheme, not all bursts will be received errorfree, strongly depending on the present interferences. The interference power is determined by the number of simultaneous transmissions and the mobile terminals' positions. With an increasing number of simultaneously transmitting terminals the carrier-to-interference ratio (C/I) decreases and the correct reception of a burst becomes less likely. Since terminals may collide a collision resolution algorithm has to be applied, which should make use of the enhanced reception properties of the system. Ward and Compton have investigated the combination of SDMA and slotted ALOHA [6]; we consider in addition splitting algorithms [3] and analyze their performance.

Fig. 2 illustrates the principle of binary splitting. All terminals belong to a so called waiting set. After accessing the radio channel they form a collision set, which is partitioned in each subsequent step (MAC frame) according to the splitting order $q$. A random experiment determines the terminals' assignment to the subsequent set. For the binary case the algorithm is also called coin flip splitting. Since the splitting algorithms are blocking algorithms new terminals are not allowed to join an ongoing collision resolution and another collision resolution instance is used in parallel. Due to the enhanced reception features of SDMA systems each collision resolution step is divided into two substeps:

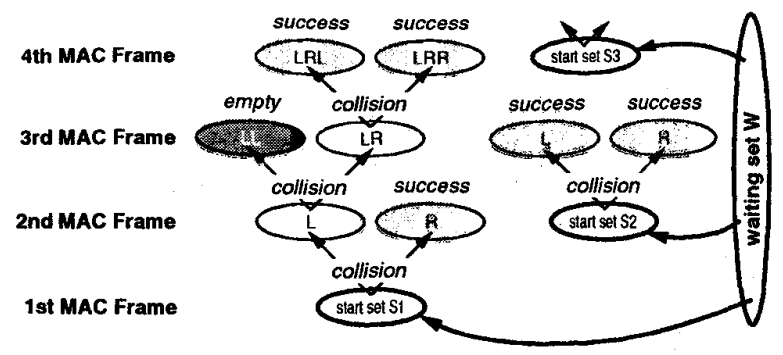

Figure 2: Parallel binary splitting

Reception substep: During the reception substep the receiver is able to receive $i$ out of $k$ bursts correctly with $0 \leq i \leq$ $\min (k, M)$. $^{\prime}$

Splitting substep: If not all $k$ bursts have been received errorfree, the collision of the remaining $k-i$ terminals has to be resolved by partitioning the collision set.

In the following, we derive an analytical model to analyze the performance of the splitting algorithm for different values of the splitting order $q$. Furthermore we describe and evaluate an adaptive algorithm that determines the sequence of the reception and splitting substeps according to the outcome of each previous step.

\section{RACH MODEL}

To investigate the presented collision resolution algorithms in the context of the described MAC protocol we use a model of the RACH derived in [7]. The model makes the following assumptions:

- A MAC frame of duration $\tau_{p}$ is able to offer any number of RACH slots.

- At the beginning of each frame the base station determines the assignment of slots to groups of terminals.

- At the end of each frame error-free feedbacks are broadcast. Deviating from [7] these feedbacks contain im this paper the information which terminals have been received correctly.

\section{COLLISION RESOLUTION BY Q-ARY SPLITTING}

\section{A. Derivation of the analytical model}

Following the mathematical analysis of [4] $p_{d}$ describes the probability of the correct decoding of a burst. Obviously $p_{d}$ is a function of the number of simultaneously transmitting terminals $k$ since $p_{d}$ decreases with decreasing $\mathrm{C} / \mathrm{I}$. We assume the same probability $p_{d}(k)$ for all terminals, neglecting the individual terminal position. Thus the probability of a correct reception of $i$ out of $k$ terminals can be described by a binomial distributed random variable $p_{k}(i)$ :

$$
p_{k}(i)=\left(\begin{array}{l}
k \\
i
\end{array}\right) p_{d}(k)^{i}\left[1-p_{d}(k)\right]^{k-i} \quad: \quad k>0
$$

Fig. 3 shows a reception step and a subsequent binary splitting step $(q=2)$ for $k=3$. Furthermore the corresponding probabilities of the correct reception of $i=0,1, \ldots, 3$ terminals and

\footnotetext{
${ }^{1}$ For $k>1$ and $i=1$ this is equivalent to capture in omnidirectional systems.
} 


$$
\begin{aligned}
& A(k, i)=\left(\begin{array}{l}
k \\
i
\end{array}\right) p_{d}(k)^{(k-i)}\left[1-p_{d}(k)\right]^{i} \\
& N_{q}(k)= \begin{cases}1+\sum_{i=0}^{k} A(k, i) N_{q}^{*}(i) & : 0<k \leq M \\
1+\sum_{i=k-M}^{k} A(k, i) N_{q}^{*}(i)+\left[1-\sum_{i=k-M}^{k} A(k, i)\right] N_{q}^{*}(k-M) & : k>M \\
1 & : k=0\end{cases}
\end{aligned}
$$

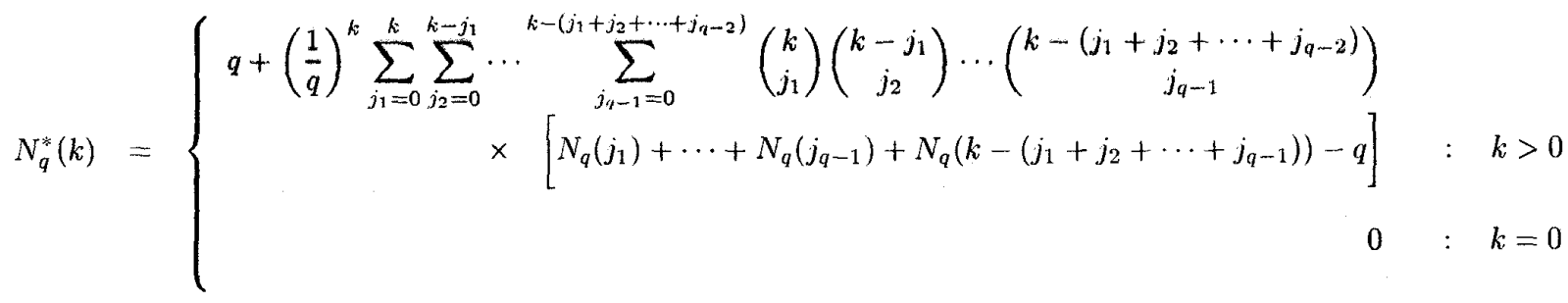

the probabilities of the possible outcomes of the splitting step are given, which have been used to derive the equations given in [4]. These equations have been generalized and different splitting orders $q$ have been incorporated. The recursive equations (3) and (4) give the mean number of slots $N_{q}(k)$ necessary to resolve a collision of $k$ terminals. Eq. (3) incorporates the case that $k>M$. The first sum gives the mean number of slots weighted by the probabilities of the cases that one or more terminals of the collision set have been received successfully. The second expression gives the mean number of slots necessary to resolve the surely remaining collision set.

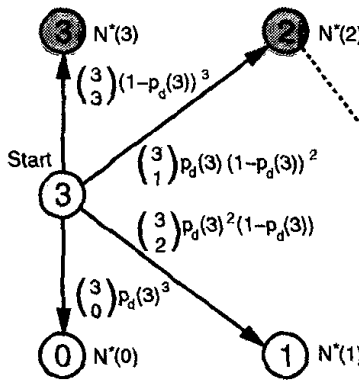

Reception step

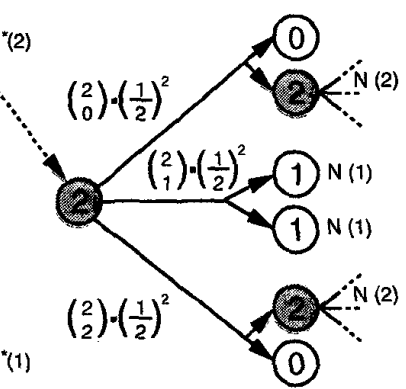

Splitting step
Figure 3: Reception step for $k=3$ and splitting step for $k=2$

\section{B. The function $p_{d}(k)$}

To evaluate and compare the different algorithms the analytical model has to be parameterized, i.e. the function $p_{d}(k)$ has to be determined. Fig. 4 shows values of $p_{d}(k)$ for $M=4$ obtained by simulating the transmission system in a pico-cellular multipath propagation environment described in [5]. Although an 8 element antenna array has been considered, spatial smoothing applied to identify additional coherent signals, reduces the upper bound $M$ of access requests that can concurrently be received to 4 . However, in the scope of this paper this function serves as an example

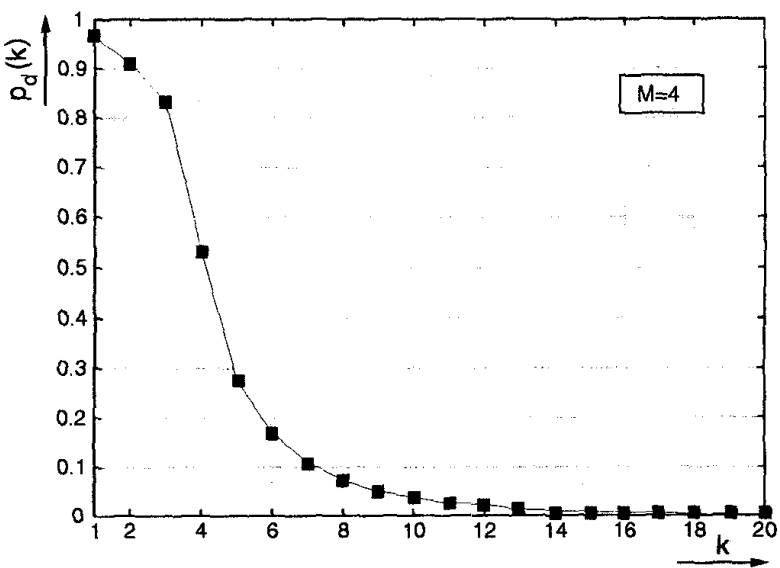

Figure 4: Reception probabilities $p_{d}(k)$ over number of transmitting terminals

and can easily be replaced by values obtained for other antenna configurations.

\section{Throughput of q-ary Splitting}

Fig. 5 shows the throughput of the RACH-P normalized by the mean number of slots $N_{q}(k)$ necessary to resolve the collision for $p_{d}(k)$ displayed in Fig. 4 . The curves have been obtained from Eqs. (3) and (4) for $q=2,3,4$. In [4] the explicit formulas for $q=2$ are given. For higher values of $q(q>4)$ the calculation becomes intractable.

\section{COLLISION RESOLUTION BY ADAPTIVE SPLITTING}

Since SDMA offers the possibility to simultaneously receive more than one signal this property can be exploited for collision resolution. We have studied the performance of the approach without collision resolution through splitting but with repeated access attempts only. But the throughput and delay curves given 


$$
N_{2, \gamma}(k)=\left\{\begin{aligned}
1 & : \quad k=0 \\
1+\sum_{i=0}^{k} A(k, i) N_{2}^{*}(i) & : \quad 0<k \leq \gamma \\
1+\sum_{i=0}^{k-\gamma-1} A(k, i) N_{2, \gamma}(i)+\sum_{i=k-\gamma}^{k} A(k, i) N_{2}^{*}(i) & : \quad \gamma<k \leq M \\
1+\sum_{i=k-M}^{k-\gamma-1} A(k, i) N_{2, \gamma}(i)+\sum_{i=k-\gamma}^{k} A(k, i) N_{2}^{*}(i) & \\
+\left[1-\sum_{i=k-M}^{k} A(k, i)\right] N_{2, \gamma}(k-M) & : \quad k>M
\end{aligned}\right.
$$

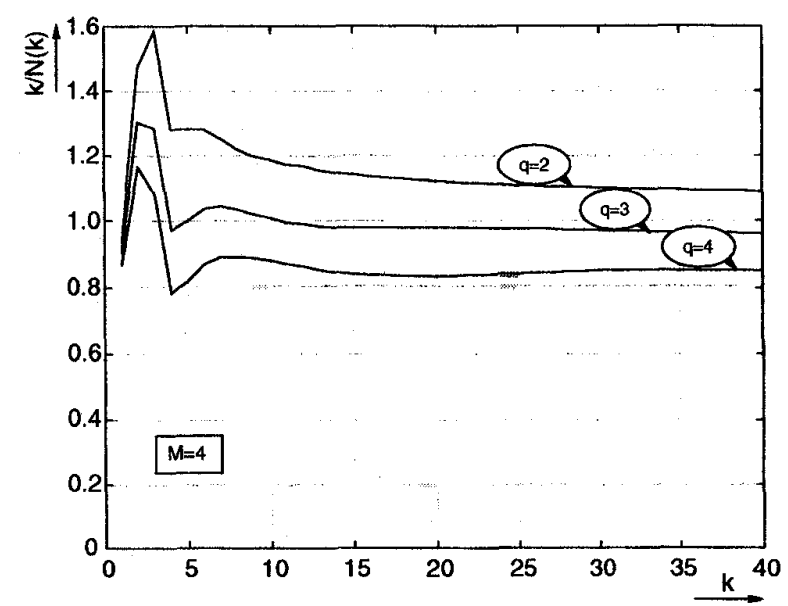

Figure 5: Throughput for q-ary Splitting with $k$ transmitting terminals

in Fig. 7 and 8 show the poor performance without splitting for increased $k$ and motivate to use additional protocol control functions. The basic idea for the adaptive algorithm investigated in the following is the dependence of the subsequent substep from the previous step's outcome. If the number of correctly received terminals $i$ remains below or equals a certain threshold $\gamma$ the algorithm continues with a binary splitting substep otherwise another reception step is performed. Fig. 6 illustrates the principle for $k=3$ and $\gamma=1$.

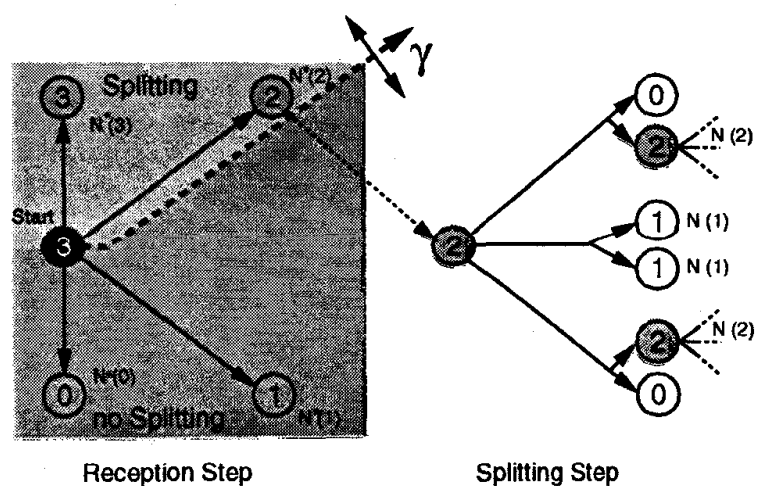

Figure 6: Adaptive Splitting with parameter $\gamma$

\section{A. Throughput of Adaptive Splitting}

The throughput for the adaptive splitting algorithm was calculated using Eq. (5), which incorporates the parameter $\gamma$. Eq. (4) for $q=2$ gives the mean number $N_{q}^{*}(k)$ of slots necessary for the binary splitting substep. Fig. 7 compares the throughput for different values of $\gamma$ and considers the curves for "no splitting" and pure binary splitting. The values are obtained using the function $p_{d}(k)$ of Fig. 4.

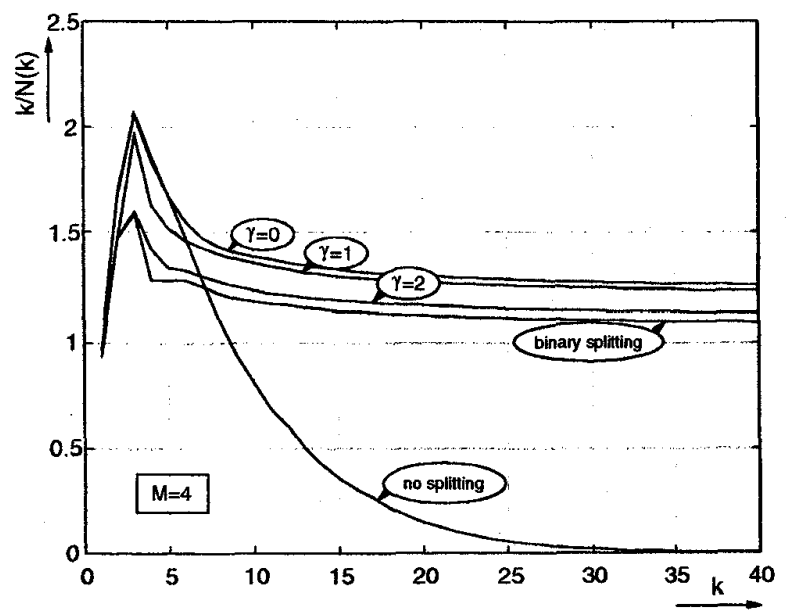

Figure 7: Throughput for adaptive Splitting with $k$ transmitting terminals

\section{B. Delay of Adaptive Splitting}

To calculate the complementary distribution function (cdf) of the theoretically unbounded delay, the probability $p_{q}(k, l, m)$ of a remaining collision set of $m$ terminals after the duration of $l$ MAC frames is derived starting with a collision set of $k$ terminals. Eq. (7) gives the probability $p_{2, \gamma}^{*}(r, m)$ that after a single reception and a single subsequent splitting step a collision set of $m$ terminals is remaining, starting with $r$ terminals.

$\hat{p}_{q}(k, l, m)=\left\{\begin{aligned} 1 & : l=0, k=m \\ 0 & : l=0, k \neq m \\ \sum_{i=0}^{k-m} \hat{p}_{q}(k, l-1, k-i) & \\ \times \quad p_{q, \gamma}^{*}(k-i, m) & : l>0\end{aligned}\right.$ 


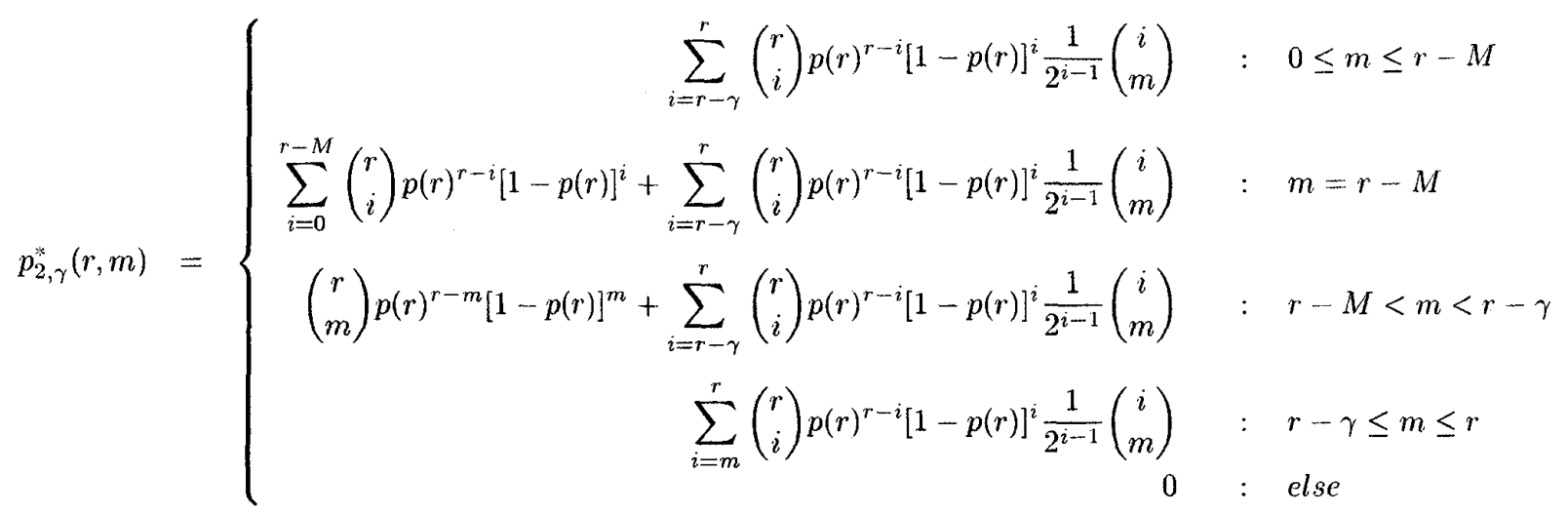

Taking into account that the last step is always a reception step, the probability of $m$ out of $k$ terminals remaining in the collision set after $l$ substeps is given by:

$$
p_{q}(k, l, m)=\sum_{i=m}^{k} \hat{p}_{q}(k, l, i)\left(\begin{array}{c}
i \\
m
\end{array}\right) p_{d}(i)^{i-m}\left[1-p_{d}(i)\right]^{m}(8)
$$

The values of the cdf of delay are equivalent to the expected number of terminals that after $l$ reception and splitting substeps, each performed during one MAC frame, still belong to the collision set:

$$
P_{q}(k, l)=\frac{1}{k} \sum_{m=0}^{k} m \cdot p_{q}(k, l, m)
$$

Finally this leads to the desired cdf with $\tau_{p}$ describing the length of the MAC frame:

$$
P_{\text {delay }, q}\left(k, \tau_{d}>\tau\right)=\left\{\begin{array}{r}
P_{q}\left(k,\left\lfloor\tau / \tau_{p}\right\rfloor-1\right): \tau \geq \tau_{p} \\
1: \tau<\tau_{p}
\end{array}\right.
$$

Fig. 8 compares the delays for the different algorithms. Note that the curves for pure binary splitting and $\gamma=2$ fall on top of each other.

\section{CONCLUSIONS}

Our analysis gives the performance of collision resolution algorithms based on splitting, incorporating the properties of enhanced signal processing and adaptive array antennas. The investigations have been performed for packet oriented systems, such as HIPERLAN/2. It is shown that collision resolution can benefit from the signal processing features in terms of throughput and delay. The adaptive algorithm introduced combines signal processing features and protocol control measures. The parameters of this algorithm allow the optimization for throughput or for delay. Also a trade-off between both of these requirements is possible. This scheme could be applied to W-ATM links to reach a desired QoS, if the associated terminals, e.g., can be divided into two groups supporting realtime and non-realtime services.

\section{References}

[1] D. Petras and U. Vornefeld, "Joint Performance of DSA++ MAC Protocol and SR/D-ARQ Protocol for wireless ATM under realistic

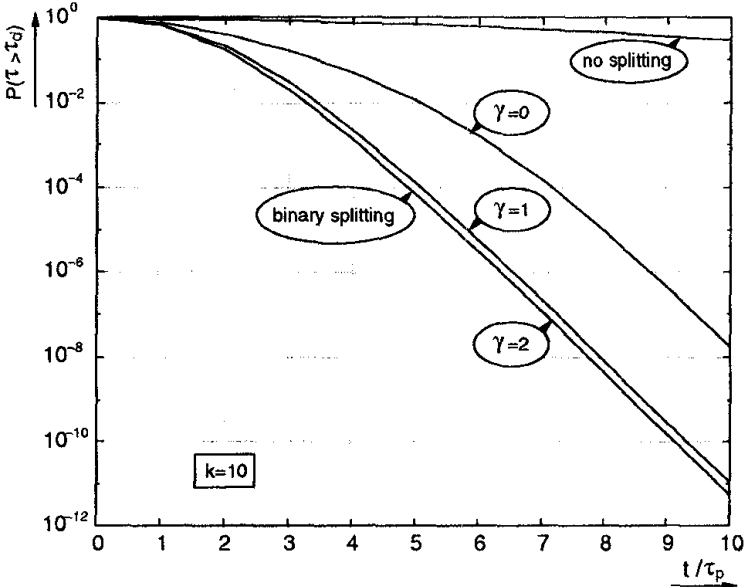

Figure 8: Cdf of Delay for $k=10$ and $M=4$

traffic and channel models," in Ist wmATM'98, (Hangzhou, China), April 1998.

[2] ETSI BRAN, "HIgh PErformance Radio Local Area Network (HIPERLAN), Requirements and Architectures for Wireless ATM Access and Interconnection," TR 101 031, ETSI, April 1997.

[3] D. Bertsekas and R. Gallager, Data Networks. Englewood Cliffs, NJ: Prentice-Hall, 1987.

[4] U. Vornefeld and E. Weltersbach, "Fast Collision Resolution with SDMA for the DSA++ MAC-Protocol for Wireless ATM Networks," in PIMRC, (Boston, MA, USA), pp. 158-162, Sept. 1998.

[5] U. Vornefeld, and Ch. M. Walke, "SDMA-Techniques for Wireless ATM - A System-oriented View," in EPMCC'99, (Paris, France), March 1999.

[6] J. Ward and R. T. Compton, "High Throughput Slotted ALOHA Packet Radio Networks with Adaptive Arrays," IEEE Transactions on Communications, vol. 41, no. 3, pp. 460-470, 1993.

[7] D. Petras and A. Krämling, "Fast Collision Resolution in Wireless ATM Networks," in 2nd MATHMOD, (Vienna, Austria), pp. 517524, Feb. 1997 